\title{
Contractibility and fixed point property: the case of Khalimsky topological spaces
}

\section{Sang-Eon $\operatorname{Han}^{*}$}

"Correspondence: sehan@jbnu.ac.kr
Institute of Pure and Applied
Mathematics, Department of
Mathematics Education, Chonbuk
National University, Jeonju-City,
Jeonbuk 54896, Republic of Korea

\begin{abstract}
Based on the notions of both contractibility and local contractibility, many works were done in fixed point theory. The present paper concerns a relation between digital contractibility and the existence of fixed points of digitally continuous maps. In this paper, establishing a new digital homotopy named by a K-homotopy in the category of Khalimsky topological spaces, we prove that in digital topology, whereas contractibility implies local contractibility, the converse does not hold. Furthermore, we address the following problem, which remains open. Let $X$ be a Khalimsky $(K$ - for short) topological space with $K$-contractibility. Then we may pose the following question: does the space $X$ have the fixed point property (FPP)? In this paper, we prove that not every $K$-topological space with $K$-contractibility has the FPP.
\end{abstract}

MSC: $55 \mathrm{~N} 35 ; 68 \mathrm{U} 10$

Keywords: Schauder's fixed point theorem; fixed point property; contractibility; local contractibility; Khalimsky homotopy; Khalimsky topology

\section{Introduction}

It is well known that Schauder's fixed point theorem [1] implies that a nonempty compact convex subset $X$ of a Banach space has a fixed point for any continuous self-map of $X$. Before referring to the work, first of all, we need to recall that a topological space $X$ has the $F P P$ if every continuous self-map $f$ of $X$ has a point $x \in X$ such that $f(x)=x$. Since every singleton obviously has the $F P P$, in studying the $F P P$ of spaces, all spaces $X$ (resp. digital images $(X, k))$ are assumed to be connected (resp. $k$-connected) with $|X| \geq 2$. In relation to the Lefschetz and Borsuk fixed point theorems $[2,3]$, there was the following conjecture [3]: let $X$ be a contractible and locally contractible space.

Then it has the $F P P$ for compact mappings.

Borsuk [2] proved that this conjecture is true in finite-dimensional metric spaces. Besides, various cases of the conjecture were proved by Cellina [4] and Fryszkowski [5]. As referred in (1.1), the contractibility of a space $X$ plays an important role in studying the FPP of $X$ and its applications. Thus, many works $[2,4-8]$ associated with contractibility are well developed.

Digital topology has a focus on studying digital topological properties of $n \mathrm{D}$ digital spaces, whereas Euclidean topology deals with topological properties of subspaces of the

(c) $2016 \mathrm{Han}$. This article is distributed under the terms of the Creative Commons Attribution 4.0 International License (http://creativecommons.org/licenses/by/4.0/), which permits unrestricted use, distribution, and reproduction in any medium, provided you give appropriate credit to the original author(s) and the source, provide a link to the Creative Commons license, and indicate if changes were made. 
$n \mathrm{D}$ real space, which has contributed to the study of some areas of computer sciences such as computer graphics, image processing, approximation theory, mathematical morphology, optimization theory, and so forth [9-13]. To study digital spaces (see Definition 1), first of all, we have often followed the method established by Rosenfeld [14], the socalled graph theoretical approach (i.e., the Rosenfeld model) $[9,11-15]$, which is proceeded in many works. Second, one of the well-studied areas is a $K$-topological space [16-18]. A number of properties of the Khalimsky $n \mathrm{D}$ space have been also used to study digital spaces $[15,16,19]$. Finally, we have used Marcus-Wyse ( $M$ - for short) topology [20-22] to study only 2D digital images.

The present paper develops a $K$-topological version of the conjecture (1.1) and some related works posed by Borsuk. At this moment, we need to recall the following differences between metric-based fixed point theory and $K$-topology-based fixed point theory. A $K$-topological space is not a metric space (see Remark 2.3), contrary to the assumption required by Borsuk. Furthermore, unlike the difference between contractibility and local contractibility in classical mathematics, the present paper proves that their digital versions have their own features (see Theorem 4.6).

In digital topology, there are several types of contractibilities associated with the corresponding digital homotopies $[9,11,17,21,23]$. After developing a $K$-homotopy, we prove that whereas in $K$-topology contractibility implies local contractibility, the converse does not hold. Similarly, we prove that whereas $k$-contractibility of a digital image $(X, k)$ implies local $k$-contractibility, the converse does not hold.

Rosenfeld (see Theorems 3.3 and 4.1 of [14]) first proved that (for more details, see [24-26])

a digital image $(X, k)$ with $|X| \geq 2$ does not have the $F P P$.

This means that only a singleton has the FPP in digital topology in a graph-theoretical approach. Nevertheless, Ege and Karaca [27] recently studied the property (1.2) in a graphtheoretical approach (see Theorem 3.8 of [27]). However, the result is proved invalid [25, $26,28]$ (see Remark 5.2). Furthermore, to formulate a digital version of the ordinary Lefschetz fixed point theorem in [27], the authors of [27] used digital homology groups of digital images in [27]. However, it turns out that almost of the assertions in [27] are incorrect $[24,26]$ because the digital version of the Lefschetz number in [27] is not a digital homotopy invariant [26]. Besides, Han [25, 26, 28] recently gave counterexamples to refute this assertion (see Remark 5.2).

Hence, in this paper, we will mainly focus ourselves on studying the $F P P$ of $K$-topological spaces instead of digital images $(X, k)$. Besides, we deal only with finite $K$-topological spaces (or compact spaces), and we can propose a digital version of (1.1) as a conjecture because contractibility implies local contractibility in digital topology (see Theorem 4.6) as follows: let $X$ be a $K$-topological space with $K$-contractibility.

Then it has the $F P P$ for $K$-continuous mappings.

To address the conjecture (1.3), the present paper proves that $K$-contractibility of a finite $K$-topological space need not imply the existence of fixed points of $K$-continuous maps (see Theorems 5.4 and 5.8). 
The rest of the paper is organized as follows. Section 2 provides basic notions and terminology from digital topology. Section 3 develops a new digital homotopy named by a $K$-homotopy to study $K$-contractibility. Section 4 investigates various properties of contractibilities in digital topology and compares them. Besides, we develop a digital version of local contractibility and prove that whereas contractibility implies local contractibil$i t y$, the converse does not hold. Section 5 proves that not every $K$-topological space with $K$-contractibility has the $F P P$, which is negative to the conjecture (1.3). But a simple $K$-path has the FPP satisfying the property (1.3). Section 6 concludes the paper with summary and further works.

\section{Preliminaries}

Let $\mathbf{Z}, \mathbf{N}$, and $\mathbf{Z}^{n}$ represent the sets of integers, natural numbers, and points in the Euclidean $n \mathrm{D}$ space with integer coordinates, respectively. Herman [29] gave the following:

Definition 1 [29] A digital space is a pair $(X, \pi)$, where $X$ is a nonempty set, and $\pi$ is a binary symmetric relation on $X$ such that $X$ is $\pi$-connected.

In Definition 1, we say that $X$ is $\pi$-connected if for any two elements $x$ and $y$ of $X$, there is a finite sequence $\left(x_{i}\right)_{i \in\left[0, l_{Z}\right.}$ of elements in $X$ such that $x=x_{0}, y=x_{l}$, and $\left(x_{j}, x_{j+1}\right) \in \pi$ for $j \in[0, l-1]_{\mathbf{Z}}$.

Remark 2.1 In Definition 1, we can consider the relation $\pi$ according to the situation such as the digital $k$-adjacency relation of (2.1) below and the $K$-adjacency relation of Definition 2 , which are both symmetric relations.

As referred in (1.3), owing to the property (1.2), the present paper mainly studies the $F P P$ from the viewpoint of $K$-topology. First, to study the property (1.3), let us recall basic notions and terminology from digital topology such as $k$-adjacency relations of $n \mathrm{D}$ integer grids, a digital $k$-neighborhood, digital continuity, and so forth [11-15]. As a generalization of digital $k$-connectivity of $\mathbf{Z}^{n}, n \in\{1,2,3\}$ [12,13], we will say that two distinct points $p, q \in \mathbf{Z}^{n}$ are $k$-adjacent (or $k(m, n)$-adjacent) if they satisfy the following property [11] (see also [20,30]):

For a natural number $m, 1 \leq m \leq n$, two distinct points

$$
p=\left(p_{1}, p_{2}, \ldots, p_{n}\right) \text { and } q=\left(q_{1}, q_{2}, \ldots, q_{n}\right) \in \mathbf{Z}^{n},
$$

are $k(m, n)$-adjacent ( $k$-adjacent for brevity) if

at most $m$ of their coordinates differ by \pm 1 , and the other coincide.

Concretely, these $k(m, n)$-adjacency relations of $\mathbf{Z}^{n}$ are determined according to the number $m \in \mathbf{N}$ [11] (see also [30]).

In terms of the operator (2.1), the $k$-adjacency relations of $\mathbf{Z}^{n}$ are obtained [11] (see also $[17,30])$ as follows:

$$
k:=k(m, n)=\sum_{i=n-m}^{n-1} 2^{n-i} C_{i}^{n},
$$

where $C_{i}^{n}=\frac{n !}{(n-i) !: i !}$. 
For a $k$-adjacency relation of $\mathbf{Z}^{n}$, a simple $k$-path with $l+1$ elements in $\mathbf{Z}^{n}$ is assumed to be an injective sequence $\left(x_{i}\right)_{i \in[0,]_{\mathbf{Z}}} \subset \mathbf{Z}^{n}$ such that $x_{i}$ and $x_{j}$ are $k$-adjacent if and only if $|i-j|=1$ [12]. If $x_{0}=x$ and $x_{l}=y$, then the length of the simple $k$-path, denoted by $l_{k}(x, y)$, is the number $l$. We say that a digital image $(X, k)$ is $k$-connected if for any two points in $X$, there is a $k$-path in $X$ connecting these two points. A simple closed $k$-curve with $l$ elements in $\mathbf{Z}^{n}$, denoted by $S C_{k}^{n, l}[11,12]$ (see Figure $1(\mathrm{a})$ ), is the simple $k$-path $\left(x_{i}\right)_{i \in[0, l-1]_{\mathbf{Z}}}$, where $x_{i}$ and $x_{j}$ are $k$-adjacent if and only if $|i-j|=1(\bmod l)$ [12] (see Figure 1$)$.

Rosenfeld [13] called a set $X \subset \mathbf{Z}^{n}$ with a $k$-adjacency a digital image and denoted it by $(X, k)$. By using the $k$-adjacency relations of $\mathbf{Z}^{n}$ of (2.2) we say that a digital $k$-neighborhood of $p$ in $\mathbf{Z}^{n}$ is the set [13] $N_{k}(p):=\{q \mid p$ is $k$-adjacent to $q\}$. Furthermore, we often use the notation [12]

$$
N_{k}^{*}(p):=N_{k}(p) \cup\{p\}
$$

For a digital image $(X, k)$, as a generalization of $N_{k}^{*}(p)$ [12], the digital $k$-neighborhood of $x_{0} \in X$ with radius $\varepsilon$ is defined in $X$ to be the following subset [11] of $X$ :

$$
N_{k}\left(x_{0}, \varepsilon\right):=\left\{x \in X \mid l_{k}\left(x_{0}, x\right) \leq \varepsilon\right\} \cup\left\{x_{0}\right\},
$$

where $l_{k}\left(x_{0}, x\right)$ is the length of the shortest simple $k$-path in $X$ from $x_{0}$ to $x$, and $\varepsilon \in \mathbf{N}$. Concretely, for $X \subset \mathbf{Z}^{n}$, we obtain [11]

$$
N_{k}(x, 1)=N_{k}^{*}(x) \cap X
$$

Second, let us now briefly recall some basic facts and terms related to $K$-topology. Motivated by the Alexandroff space [31], the Khalimsky line topology on $\mathbf{Z}$ is induced by the set $\left\{[2 n-1,2 n+1]_{\mathbf{Z}}: n \in \mathbf{Z}\right\}$ as a subbase [31], where for two distinct points $a$ and $b$ in $\mathbf{Z},[a, b]_{\mathbf{Z}}=\{n \in \mathbf{Z} \mid a \leq n \leq b\}[9,12]$. Furthermore, the product topology on $\mathbf{Z}^{n}$ induced by $(\mathbf{Z}, \kappa)$ is called the Khalimsky product topology on $\mathbf{Z}^{n}$ (or Khalimsky $n D$ space), which is denoted by $\left(\mathbf{Z}^{n}, \kappa^{n}\right)$. A point $x=\left(x_{1}, x_{2}, \ldots, x_{n}\right) \in \mathbf{Z}^{n}$ is pure open if all coordinates are odd; and it is pure closed if each of the coordinates is even [16]. The other points in $\mathbf{Z}^{n}$ are called mixed [16].

For a point $p:=\left(p_{1}, p_{2}\right)$ in $\left(\mathbf{Z}^{2}, \kappa^{2}\right)$, its smallest open neighborhood $\mathrm{SN}_{K}(p)$ is obtained [16]:

$$
\mathrm{SN}_{K}(p):=\left\{\begin{array}{l}
\{p\} \text { if } p \text { is pure open, } \\
\left\{\left(p_{1}-1, p_{2}\right), p,\left(p_{1}+1, p_{2}\right)\right\} \text { if } p \text { is closed-open, } \\
\left\{\left(p_{1}, p_{2}-1\right), p,\left(p_{1}, p_{2}+1\right)\right\} \text { if } p \text { is open-closed, } \\
N_{8}^{*}(p) \text { if } p \text { is pure closed, }
\end{array}\right\}
$$

where the point $p:=\left(p_{1}, p_{2}\right)$ is called closed-open (resp. open-closed) if $p_{1}$ is even (resp. odd) and $p_{2}$ is odd (resp. even).

In this paper, each space $X \subset \mathbf{Z}^{n}$ related to $K$-topology is considered to be a subspace $\left(X, \kappa_{X}^{n}\right)$ induced by $\left(\mathbf{Z}^{n}, \kappa^{n}\right)[16,20]$.

Let us now recall the structure of $\left(\mathbf{Z}^{n}, \kappa^{n}\right)$. In each of the spaces of Figures 1-9, a black jumbo dot means a pure open point, and further, the symbols $\mathbf{\square}$ and $\bullet$ mean a pure closed 
(a)
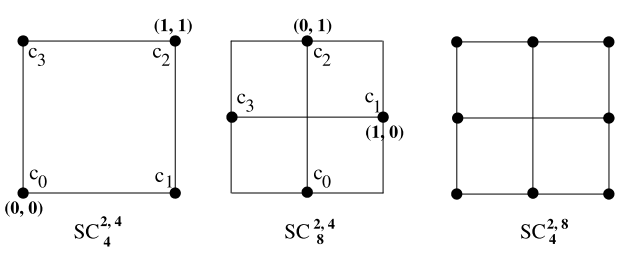

(b)
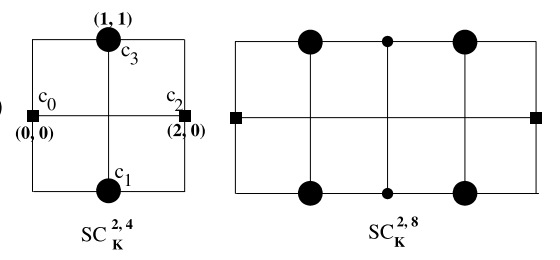

Figure 1 Simple closed curves. (a) $S C_{4}^{2,4}[27], S C_{8}^{2,4}[9]$, and $S C_{4}^{2,8}[11] ;$ (b) $S C_{K}^{2,4}$ and $S C_{K}^{2,8}[30]$.

point and a mixed point, respectively. In relation to the further statement of a pure point and a mixed point, we can say that a point $x$ is open if $\mathrm{SN}_{K}(x)=\{x\}$, where $\mathrm{SN}_{K}(x)$ means the smallest neighborhood of $x \in \mathbf{Z}^{n}$. Many studies have examined various properties of a $K$-continuous map, connectedness, $K$-adjacency, a $K$-homeomorphism $[16,17,20]$.

Let us recall the following notions for studying $K$-topological spaces.

Definition 2 [20] Let $\left(X, \kappa_{X}^{n}\right):=X$ be a $K$-topological space. We say that two distinct points $x, y \in X$ are $K$-adjacent if $x \in \mathrm{SN}_{K}(y)$ or $y \in \mathrm{SN}_{K}(x)$. Then we define the following:

We say that a $K$-path from $x$ to $y$ in $X$ is a sequence $(x)_{i \in\left[0, l_{Z}\right.}, l \geq 2$, in $X$ such that $x_{0}=x$, $x_{l}=y$ and each point $x_{i}$ is $K$-adjacent to $x_{i+1}$ and $i \in[0, l]_{\mathrm{Z}}$. The number $l$ is called the length of this path. A simple $K$-path in $X$ is the injective sequence $\left(x_{i}\right)_{i \in\left[0, l_{\mathbf{Z}}\right.}$ such that $x_{i}$ and $x_{j}$ are $K$-adjacent if and only if $|i-j|=1$.

Furthermore, we say that a simple closed $K$-curve with $l$ elements in $\mathbf{Z}^{n}$, denoted by $S C_{K}^{n, l}, l \geq 4$, is a simple $K$-path $\left(x_{i}\right)_{i \in[0, l-1]_{\mathbf{Z}}}$, where $x_{i}$ and $x_{j}$ are $K$-adjacent if and only if $|i-j|=1(\bmod l)$.

Example 2.2 In Figure 1(a), $S C_{4}^{2,4}, S C_{8}^{2,4}$, and $S C_{4}^{2,8}$ are shown. In Figure 1(b), we have $S C_{K}^{2,4}$ and $S C_{K}^{2,8}$.

Remark 2.3 Each $K$-topological space is not a metric space because it is neither a $T_{1}$-space nor a regular space although it has a countable basis (see the property (2.5)). Besides, in case we follow a graph-theoretical approach for studying digital spaces (or digital images), a mapping between digital spaces is a graph homomorphism instead of a topological (compact) mapping.

\section{Development of a Khalimsky homotopy and its properties}

This section firstly develops the notion of a $K$-homotopy and investigates various properties of a $K$-homotopy, which will be used to study both contractibility and local contractibility from the viewpoint of digital topology in Sections 3 and 4. Let us now recall some properties of digital spaces in a graph-theoretical approach. To map every $k_{0}$-connected subset of $\left(X, k_{0}\right)$ into a $k_{1}$-connected subset of $\left(Y, k_{1}\right)$, the paper [13] established the notion of digital continuity of maps between digital images. Motivated by this 
approach, the digital continuity of maps between digital images was represented as follows.

Proposition 3.1 $[11,15]$ Let $\left(X_{i}, k_{i}\right)$ be digital images in $\mathbf{Z}^{n_{i}}$ with the $k_{i}$-adjacency relations of (2.2), $i \in\{0,1\}$. A function $f:\left(X_{0}, k_{0}\right) \rightarrow\left(X_{1}, k_{1}\right)$ is $\left(k_{0}, k_{1}\right)$-continuous if and only if $f\left(N_{k_{0}}(x, 1)\right) \subset N_{k_{1}}(f(x), 1)$ for every $x \in X_{0}$.

In Proposition 3.1, in case $k_{1}=k_{2}$, the map $f$ is called a $k_{1}$-continuous map. By using this concept we establish a digital topological category, denoted by $D T C$, consisting of two sets [11] (see also [30]):

- for any set $X \subset \mathbf{Z}^{n}$, the set of $(X, k)$ in $\mathbf{Z}^{n}$ as objects of $D T C$;

- for every ordered pair of objects $\left(X_{i}, k_{i}\right), i \in\{1,2\}$, the set of all $\left(k_{0}, k_{1}\right)$-continuous maps as morphisms of $D T C$.

In $D T C$, in case $k_{0}=k_{1}:=k$, we will particularly use the notation $\mathrm{DTC}(k)$ [21].

Based on the pointed digital homotopy in $[9,16]$, the following notion of a $k$-homotopy relative to a subset $A \subset X$ is often used to study a $k$-homotopic thinning and to classify digital images $(X, k)$ in $\mathbf{Z}^{n}[17,30]$.

Definition 3 [11] (see also [15]) Let $\left((X, A), k_{0}\right)$ and $\left(Y, k_{1}\right)$ be a digital image pair and a digital image, respectively. Let $f, g: X \rightarrow Y$ be $\left(k_{0}, k_{1}\right)$-continuous functions. Suppose that there exist $m \in \mathbf{N}$ and a function $F: X \times[0, m]_{\mathrm{Z}} \rightarrow Y$ such that

$(\bullet 1)$ for all $x \in X, F(x, 0)=f(x)$ and $F(x, m)=g(x)$;

$(\bullet 2)$ for all $x \in X$, the induced function $F_{x}:[0, m]_{\mathrm{Z}} \rightarrow Y$ given by $F_{x}(t)=F(x, t)$ for all $t \in[0, m]_{\mathbf{Z}}$ is $\left(2, k_{1}\right)$-continuous;

$(\bullet 3)$ for all $t \in[0, m]_{\mathrm{Z}}$, the induced function $F_{t}: X \rightarrow Y$ given by $F_{t}(x)=F(x, t)$ for all $x \in X$ is $\left(k_{0}, k_{1}\right)$-continuous.

Then we say that $F$ is a $\left(k_{0}, k_{1}\right)$-homotopy between $f$ and $g$ [9], denoted by $f \simeq_{\left(k_{0}, k_{1}\right)} g$.

(•4) Furthermore, for all $t \in[0, m]_{\mathrm{Z}}, F_{t}(x)=f(x)=g(x)$ for all $x \in A$.

Then we call $F$ a $\left(k_{0}, k_{1}\right)$-homotopy relative to $A$ between $f$ and $g$ and we say that $f$ and $g$ are $\left(k_{0}, k_{1}\right)$-homotopic relative to $A$ in $Y$, denoted $f \simeq_{\left(k_{0}, k_{1}\right) \operatorname{rel} A} g$.

In Definition 3, if $A=\left\{x_{0}\right\} \subset X$, then we say that $F$ is a pointed $\left(k_{0}, k_{1}\right)$-homotopy at $\left\{x_{0}\right\}$ [9]. In addition, if $k_{0}=k_{1}$ and $n_{0}=n_{1}$, then we say that $f$ and $g$ are pointed $k_{0}$-homotopic in $Y$. If, for some $x_{0} \in X, 1_{X}$ is $k$-homotopic to the constant map in the space $\left\{x_{0}\right\}$ relative to $\left\{x_{0}\right\}$, then we say that $\left(X, x_{0}\right)$ is pointed $k$-contractible $[9,11]$.

Remark 3.2 As for the function $F: X \times[0, m]_{\mathrm{Z}} \rightarrow Y$ of Definition 3, the Cartesian product $X \times[0, m]_{\mathbf{Z}}$ is just a set without any consideration of a digital adjacency for a Cartesian product. In other words, the set $X \times[0, m]_{\mathrm{Z}}$ is assumed to be a disjoint union $X \times\{i\}$, $i \in[0, m]_{\mathbf{Z}}$.

The following notion of a digital homotopy equivalence was firstly introduced in [10,32] to classify digital images in $D T C$. 
Definition $4[10,32]$ In $D T C$, for two digital images $\left(X, k_{0}\right)$ and $\left(Y, k_{1}\right)$, if there are a $\left(k_{0}, k_{1}\right)$-continuous map $h: X \rightarrow Y$ and a $\left(k_{1}, k_{0}\right)$-continuous map $l: Y \rightarrow X$ such that $l \circ h$ is $k_{0}$-homotopic to $1_{X}$ and $h \circ l$ is $k_{1}$-homotopic to $1_{Y}$, then the map $h: X \rightarrow Y$ is called a $\left(k_{0}, k_{1}\right)$-homotopy equivalence. In this case, we use the notation $X \simeq_{\left(k_{0}, k_{1}\right) \cdot h \cdot e} Y$. Furthermore, if $k_{0}=k_{1}$ and $n_{0}=n_{1}$, then we call $h$ a $k_{0}$-homotopy equivalence, and we use the notation $X \simeq_{k_{0} \cdot h \cdot e} Y$.

We say that a digital image $(X, k)$ is $k$-contractible if $X \simeq_{k \cdot h \cdot e}\left\{x_{0}\right\}$ for some point $x_{0} \in X$. Motivated by both the $k$-homotopy in Definition 3 and the $k$-homotopy equivalence in Definition 4, their $K$-topological versions are obtained (see Definitions 6 and 7 ) in $K$-topology. Let us now recall the $K$-continuity of maps between $K$-topological spaces. As usual, for two $K$-topological spaces $\left(X, \kappa_{X}^{n_{0}}\right):=X$ and $\left(Y, \kappa_{Y}^{n_{1}}\right):=Y$, a map $f: X \rightarrow Y$ is called continuous at a point $x \in X$ if for any open set $O_{f(x)} \subset Y$ containing the point $f(x)$, there is an open set $O_{x} \subset X$ containing the point $x$ such that $f\left(O_{x}\right) \subset O_{f(x)}$. Namely, we can represent it as

$$
f\left(\mathrm{SN}_{K}(x)\right) \subset \mathrm{SN}_{K}(f(x))
$$

because each point $x$ in a $K$-topological space $X$ always has $\operatorname{SN}_{K}(x) \subset X$.

By using spaces $\left(X, \kappa_{X}^{n}\right):=X$ and $K$-continuous maps, we have a topological category, denoted by $K T C$, consisting of the following two sets [20]:

(1) for any set $X \subset \mathbf{Z}^{n}$, the set of spaces $\left(X, \kappa_{X}^{n}\right)$ as objects of $K T C$ denoted by $\mathrm{Ob}(K T C)$;

(2) for all pairs of elements in $\mathrm{Ob}(K T C)$, the set of all $K$-continuous maps between them as morphisms.

To study $K$-topological spaces in $\mathbf{Z}^{n}$, we need to recall a $K$-homeomorphism as follows:

Definition $5[16,20]$ For two spaces $\left(X, \kappa_{X}^{n_{0}}\right):=X$ and $\left(Y, \kappa_{Y}^{n_{1}}\right):=Y$, a map $h: X \rightarrow$ $Y$ is called a $K$-homeomorphism if $h$ is a $K$-continuous bijection and $h^{-1}: Y \rightarrow X$ is $K$-continuous.

In $\left(\mathbf{Z}^{n}, T^{n}\right)$, we say that a simple closed $K$-curve with $l$ elements in $\mathbf{Z}^{n}$ is a path $\left(x_{i}\right)_{i \in[0, l-1]_{\mathbf{Z}}} \subset \mathbf{Z}^{n}, l \geq 4$, that is $K$-homeomorphic to a quotient space of a Khalimsky line interval $[a, b]_{\mathbf{Z}}$ in terms of the identification of the only two end points $a$ and $b[20]$, where both of the numbers $a$ and $b$ in $[a, b]_{\mathrm{Z}}$ are even or odd.

Since the Khalimsky $n \mathrm{D}$ topological space is a box product of the Khalimsky line space $(\mathbf{Z}, \kappa)$, we obviously obtain the following:

\section{Lemma 3.3}

(1) Put $\mathbf{Z}^{n} \times\{i\}:=\mathbf{Z}_{i}^{n}, i \in \mathbf{Z}$. Assume $\mathbf{Z}_{i}^{n}$ to be the topological space $\left(\mathbf{Z}_{i}^{n}, \kappa_{\mathbf{Z}_{i}^{n}}^{n+1}\right)$. Then for any $i, j \in 2 \mathbf{Z}$ or $\{2 n+1 \mid n \in \mathbf{Z}\}$, we see that $\left(\mathbf{Z}_{i}^{n}, \kappa_{\mathbf{Z}_{i}^{n}}^{n+1}\right)$ is K-homeomorphic to $\left(\mathbf{Z}_{j}^{n}, \kappa_{\mathbf{Z}_{j}^{n}}^{n+1}\right)$.

(2) $\left(\mathbf{Z}^{n}, \kappa^{n}\right)$ is assumed to be a proper subspace of $\left(\mathbf{Z}^{n+1}, \kappa^{n+1}\right)$ with the relative topology on $\mathbf{Z}^{n}$ induced by $\left(\mathbf{Z}^{n+1}, \kappa^{n+1}\right), n \in \mathbf{N}$.

Proof (1) Consider the map $h:\left(\mathbf{Z}_{i}^{n}, \kappa_{\mathbf{Z}_{i}^{n}}^{n+1}\right) \rightarrow\left(\mathbf{Z}_{j}^{n}, \kappa_{\mathbf{Z}_{j}^{n}}^{n+1}\right)$ given by $h(x, i)=(x, j)$, where $x \in$ $\left(\mathbf{Z}_{i}^{n}, \kappa_{\mathbf{Z}_{i}^{n}}^{n+1}\right)$. Then $h$ is obviously a $K$-homeomorphism. 
(2) Considering $\mathbf{Z}^{n}$ to be $\mathbf{Z}^{n} \times\{0\} \subset \mathbf{Z}^{n+1},\left(\mathbf{Z}^{n}, \kappa^{n}\right)$ is assumed to be a proper subspace of $\left(\mathbf{Z}^{n+1}, \kappa^{n+1}\right)$ with the relative topology on $\mathbf{Z}^{n}$ induced by $\left(\mathbf{Z}^{n+1}, \kappa^{n+1}\right), n \in \mathbf{N}$.

By Lemma 3.3, we obtain the following:

\section{Proposition 3.4}

(1) Any K-interval $\left([a, b]_{\mathbf{Z}}, \kappa_{[a, b]_{\mathbf{Z}}}\right)$ can be embedded into a simple $K$-path in $\left(\mathbf{Z}^{n}, \kappa^{n}\right)$.

(2) $\left(X, \kappa_{X}^{n}\right)$ is equivalent to the subspace $X \times\{0\}$ of $\left(\mathbf{Z}^{n+1}, \kappa^{n+1}\right)$ up to $K$-homeomorphism.

(3) $S C_{K}^{n, l}$ is equivalent to the subspace $S C_{K}^{n, l} \times\{0\}$ of $\left(\mathbf{Z}^{n+1}, \kappa^{n+1}\right)$ up to K-homeomorphism.

(4) $S C_{K}^{n_{1}, l}$ is K-homeomorphic to $S C_{K}^{n_{2}, l}$ even if $n_{1} \neq n_{2}$.

(5) Let $X$ and $Y$ be simple $K$-paths with the same elements. Then $\left(X, \kappa_{X}\right)$ need not be K-homeomorphic to $\left(Y, \kappa_{Y}\right)$.

Proof (1) It suffices to prove that any $K$-interval $\left([a, b]_{\mathbf{Z}}, \kappa_{[a, b]_{\mathbf{Z}}}\right)$ is $K$-homeomorphic to a certain simple $K$-path, denoted by $\left(x_{i}\right)_{i \in[0, l]_{\mathbf{Z}}}$, in $\left(\mathbf{Z}^{n}, \kappa^{n}\right)$ such that $|b-a|=l$. Indeed, we can take a subspace $\left(x_{i}\right)_{i \in[0, l]_{\mathbf{Z}}} \subset\left(\mathbf{Z}^{n}, \kappa^{n}\right)$ that is $K$-homeomorphic to $\left([a, b]_{\mathbf{Z}}, \kappa_{[a, b]_{\mathbf{Z}}}\right)$ in terms of the mapping of $f:\left([a, b]_{\mathbf{Z}}, \kappa_{[a, b]_{\mathbf{Z}}}\right) \rightarrow\left(x_{i}\right)_{i \in[0, l]_{\mathbf{Z}}} \subset\left(\mathbf{Z}^{n}, \kappa^{n}\right)$ given by

$$
f(a)=x_{0}, \quad f(a+i)=x_{i}, \quad i \in[1, l-1]_{\mathrm{Z}}, \quad f(b)=x_{l}
$$

such that for $i, j \in[0, l]_{\mathrm{Z}}$ (see Figure $\left.2(\mathrm{a})\right)$,

$$
x_{i} \in \mathrm{SN}_{K}\left(x_{j}\right) \quad \text { or } \quad x_{j} \in \mathrm{SN}_{K}\left(x_{i}\right) \quad \text { in }\left(\mathbf{Z}^{n}, \kappa^{n}\right) \quad \text { if and only if }|i-j|=1 \text {, }
$$

$x_{i}, x_{j} \in\left(x_{i}\right)_{i \in[0, l]_{\mathbf{Z}}}:=[a, b]_{\mathbf{Z}}$.

(2) By Lemma 3.3 the proof is completed (see Figures 2(b-1), 2(b-2), and 2(c)). For instance, consider the space $\left(X, \kappa_{X}^{2}\right)$ in Figure 2(c-1). Furthermore, consider the space $\left(X \times\{0\}:=X_{0}, \kappa_{X_{0}}^{3}\right)$ in Figure $2(\mathrm{c}-2)$. Then we see that $\left(X, \kappa_{X}^{2}\right)$ is $K$-homeomorphic to $\left(X_{0}, \kappa_{X_{0}}^{3}\right)$.

(3) By Proposition 3.4(2) the proof is completed.

(4) Owing to the property of $S C_{K}^{n, l}$, there is an embedding $i: S C_{K}^{n, l} \rightarrow S C_{K}^{n, l} \times\{0\} \subset \mathbf{Z}^{n+1}$. More precisely, take any two $K$-adjacent points $x, y \in S C_{K}^{n, l}$. If $\operatorname{SN}_{K}(x) \ni y$, then we see that $\mathrm{SN}_{K}(y)=\{y\}$ and, further, $\sharp\left(\mathrm{SN}_{K}(x)\right)=3$. Since the cardinalities of $S C_{K}^{n_{1}, l}:=\left(x_{i}\right)_{i \in[0, l]_{\mathrm{Z}}}$ and $S C_{K}^{n_{2}, l}:=\left(y_{i}\right)_{i \in[0, l]_{\mathbf{Z}}}$ are equal to each other, owing to the properties of $S C_{K}^{n_{i}, l}, i \in\{1,2\}$, we obtain

$$
\left\{\begin{array}{l}
\sharp\left\{x_{i} \in S C_{K}^{n_{1}, l} \mid \sharp \mathrm{SN}_{K}\left(x_{i}\right)=3\right\}=\sharp\left\{y_{i} \in S C_{K}^{n_{2}, l} \mid \sharp \mathrm{SN}_{K}\left(y_{i}\right)=3\right\}, \\
\sharp\left\{x_{j} \in S C_{K}^{n_{1}, l} \mid \sharp \operatorname{SN}_{K}\left(x_{j}\right)=1\right\}=\sharp\left\{y_{j} \in S C_{K}^{n_{2}, l} \mid \sharp \operatorname{SN}_{K}\left(y_{j}\right)=1\right\},
\end{array}\right\}
$$

where the symbol $\sharp$ means the cardinality of a given set. Then we establish a $K$-homeomorphism between $S C_{K}^{n_{i}, l}, i \in\{1,2\}$, as follows: for the points $x_{i}, x_{j}, y_{i}$, and $y_{j}$ in (3.1), consider the mapping

$$
x_{i} \rightarrow y_{i} \quad \text { and } \quad x_{j} \rightarrow y_{j}
$$

where $x_{j} \in \mathrm{SN}_{K}\left(x_{i}\right)$ and $y_{j} \in \mathrm{SN}_{K}\left(y_{i}\right)$ if and only if $|i-j|=1$ and $i, j \in[0, l]_{\mathrm{Z}}$. Then it is obvious that the mapping of (3.2) is a $K$-homeomorphism. 
(a)

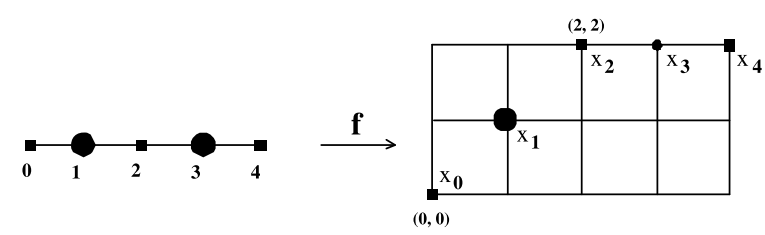

(b)
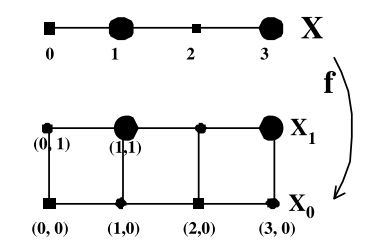

(b-1)
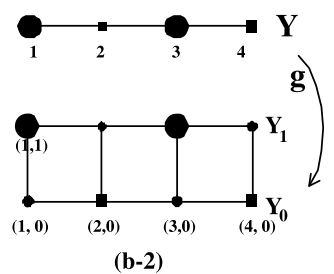

(b-2)

(d)

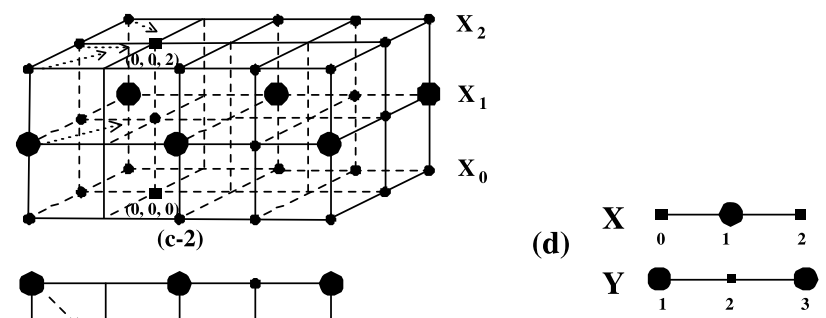

$\left(\mathbf{X}, k_{\mathbf{X}}\right)$

Figure 2 Explanation of a $\boldsymbol{K}$-homotopy. (a) Explanation of embedding a $K$-interval into $\left(\mathbf{Z}^{n} \boldsymbol{K}^{n}\right)$;

(b)-(c) a process of a $K$-homotopy; (d) comparison between two simple $K$-paths.

(5) Consider two simple $K$-paths $\left(X=[0,2]_{\mathrm{Z}}, \kappa_{X}\right)$ and $\left(Y=[1,3]_{\mathrm{Z}}, \kappa_{Y}\right)$ (see Figure $2(\mathrm{~d})$ ). Whereas $\left(X=[0,2]_{\mathrm{Z}}, \kappa_{X}\right)$ has only one singleton as a smallest open set, $\left(Y=[1,3]_{\mathrm{Z}}, \kappa_{Y}\right)$ has two singletons as smallest open sets, which cannot be $K$-homeomorphic to each other.

To develop the notion of a $K$-homotopy in $K T C$ (see Definition 6), consider two $K$-topological spaces $X:=\left(X, \kappa_{X}^{n}\right)$ and a Khalimsky interval (K-interval for short) $\left([a, b]_{\mathrm{Z}}, \kappa_{[a, b] \mathrm{Z}}\right)$. Then, depending on the given space $X$, we may consider the product space $(X \times$ $\left.[0, m]_{\mathrm{Z}}:=X^{\prime}, \kappa_{X^{\prime}}^{n+1}\right)$ or $\left(X \times[1, m+1]_{\mathrm{Z}}:=X^{\prime}, \kappa_{X^{\prime}}^{n+1}\right)$, that is, $[a, b]_{\mathbf{Z}} \in\left\{[0, m]_{\mathbf{Z}},[1, m+1]_{\mathrm{Z}}\right\}$ (see Lemma 3.3).

Let us now establish the notion of a $K$-homotopy. Furthermore, consider any $\left(X, \kappa_{X}^{n}\right)$ and $\left([a, b]_{\mathbf{Z}}, \kappa_{[a, b]_{\mathbf{Z}}}\right)$, where $[a, b]_{\mathbf{Z}} \in\left\{[0, m]_{\mathbf{Z}},[1, m+1]_{\mathbf{Z}}\right\}$. Then, by Lemma 3.3 and Proposition 3.4(2) we see that $\left(X, \kappa_{X}^{n}\right)$ is equivalent to $\left(X \times\{0\}:=X_{0}, \kappa_{X_{0}}^{n+1}\right)$ or $\left(X \times\{1\}:=X_{1}, \kappa_{X_{1}}^{n+1}\right)$ up to $K$-homeomorphism (see Figure 2(c)) or Figure 2(c-2)). Thus, we can now establish the notion of a $K$-homotopy.

Definition 6 In $K T C$, for two spaces $X:=\left(X, \kappa_{X}^{n_{0}}\right)$ and $Y:=\left(Y, \kappa_{Y}^{n_{1}}\right)$, let $f, g: X \rightarrow Y$ be $K$ continuous functions. Suppose that there exist a $K$-interval $\left([a, b]_{\mathbf{Z}}, \kappa_{[a, b]_{\mathbf{Z}}}\right)$ and a function $F: X \times[a, b]_{\mathrm{Z}} \rightarrow Y$ such that

$(* 1)$ for all $x \in X, F(x, a)=f(x)$ and $F(x, b)=g(x)$; 
$(* 2)$ for all $x \in X$, the induced function $F_{x}:\left([a, b]_{\mathrm{Z}}, \kappa_{[a, b]_{\mathrm{Z}}}\right) \rightarrow Y$ defined by $F_{x}(t)=F(x, t)$ for all $t \in\left([a, b]_{\mathbf{Z}}, \kappa_{[a, b]_{\mathbf{Z}}}\right)$ is $K$-continuous;

$(* 3)$ for all $t \in[a, b]_{\mathbf{Z}}$, the induced function $F_{t}: X \rightarrow Y$ defined by $F_{t}(x)=F(x, t)$ for all $x \in X$ is $K$-continuous.

Then we say that $F$ is a $K$-homotopy between $f$ and $g$, and $f$ and $g$ are $K$-homotopic in $Y$, denoted $f \simeq_{K} g$.

In $K T C$, we say that a $K$-topological space $X$ is $K$-contractible if the identity map $1_{X}$ is $K$-homotopic in $X$ to a constant map with the space consisting of some point $x_{0} \in X$.

Remark 3.5 (Comparison between a $k$-homotopy in DTC and a $K$-homotopy in $K T C$ )

(1) Comparing the $K$-homotopy in Definition 6 with the $k$-homotopy in DTC (see Definition 3), we find some differences between them (see Remark 3.2).

Owing to the $K$-topological structure of $X:=\left(X, \kappa_{X}^{n_{0}}\right)$, first of all, the set $X \times[0, m]_{\mathrm{Z}}$ of Definition 3 and that of Definition 6 are different from each other because the latter has the $K$-topological structure. Second, depending on the situation of $X$ in Definition 6, we need to take the number $m$ of $\left([0, m]_{\mathbf{Z}}, \kappa_{[0, m]_{\mathbf{Z}}}\right)$ even or odd, so that we do the required process under a $K$-homotopy as in Definition 6.

For instance, let us assume $\left(X, \kappa_{X}^{n_{0}}\right)$ of Definition 6 to be either $\left([0,3]_{\mathrm{Z}}, \kappa_{[0,3]_{\mathrm{Z}}}\right)$ or $\left([1,4]_{\mathbf{Z}}, \kappa_{[1,4]_{\mathbf{Z}}}\right)$. In case $\left(X, \kappa_{X}^{n_{0}}\right):=\left([0,3]_{\mathbf{Z}}, \kappa_{[0,3]_{\mathbf{Z}}}\right)$, we see that the space $[0,3]_{\mathbf{Z}} \times\{0\}:=X_{0}$ (see Figure 2(b-1)) as a subspace of $\left(\mathbf{Z}^{2}, \kappa^{2}\right)$ is $K$-homeomorphic to $\left([0,3]_{\mathbf{Z}}, \kappa_{[0,3]_{\mathbf{Z}}}\right)$ (see Figure 2(b-1)). Besides, we see that $\left(X_{0}, \kappa_{X_{0}}^{2}\right)$ is $K$-homeomorphic to $\left(X_{1}, \kappa_{X_{1}}^{2}\right)$ (see Figure 2(b-1)).

In case $\left(X, \kappa_{X}^{n_{0}}\right):=\left([1,4]_{\mathrm{Z}}, \kappa_{[1,4] \mathrm{Z}}\right)$, we see that the space $[1,4]_{\mathrm{Z}} \times\{0\}:=Y_{0}$ (see Figure $2(\mathrm{~b}-2))$ as a subspace of $\left(\mathbf{Z}^{2}, \kappa^{2}\right)$ is $K$-homeomorphic to $\left([1,4]_{\mathbf{Z}}, \kappa_{[1,4]_{\mathbf{Z}}}\right)$ (see Figure $2(\mathrm{~b}-2)$ ). Besides, we see that $\left(Y_{0}, \kappa_{Y_{0}}^{2}\right)$ is $K$-homeomorphic to $\left(Y_{1}, \kappa_{Y_{1}}^{2}\right)$ (see Figure $2(\mathrm{~b}-2))$.

(2) Consider the space $\left(X, \kappa_{X}^{2}\right)$ in Figure 2(c-1). Then, for $X \times\{i\}:=X_{i}, i \in[0,2]_{\mathrm{Z}}$, it is clear that each of the subspaces $\left(X_{i}, \kappa_{X_{i}}^{3}\right)$ is $K$-homeomorphic to $\left(X, \kappa_{X}^{2}\right)$ (see Figure 2(c-2)).

Furthermore, owing to the current version of a $K$-homotopy, the $K$-continuity of the map $F_{x}(t)=F(x, t)$ of the property $(* 2)$ holds.

(3) Consider the space $\left(X, \kappa_{X}^{2}\right)$ in Figure $3(\mathrm{c})$, where $X:=\{(0,0),(1,1),(2,1),(3,1)\}$. Then consider the transformation from $\left(X, \kappa_{X}^{2}\right)$ to $\left(Y, \kappa_{Y}^{2}\right)$ as shown in Figure $3(\mathrm{c})$, where $Y:=$ $\{(1,2),(2,3),(3,3),(4,3)\}$. Whereas the mapping cannot be a $K$-homotopy that transforms $\left(X, \kappa_{X}^{2}\right)$ onto $\left(Y, \kappa_{Y}^{2}\right)$, it can be an 8-homotopy without the $K$-topological structure.

To classify $K$-topological spaces in terms of a certain homotopy equivalence in $K T C$, we use the following:

Definition 7 In $K T C$, for two spaces $\left(X, \kappa_{X}^{n_{0}}\right):=X$ and $\left(Y, \kappa_{Y}^{n_{1}}\right):=Y$, if there are $K$-continuous maps $h: X \rightarrow Y$ and $l: Y \rightarrow X$ such that $l \circ h$ is $K$-homotopic to $1_{X}$ and $h \circ l$ is $K$-homotopic to $1_{Y}$, then the map $h: X \rightarrow Y$ is called a $K$-homotopy equivalence, denoted $X \simeq_{K \cdot h \cdot e} Y$.

We say that a digital space $\left(X, \kappa_{X}^{n}\right)$ is $K$-contractible if $X \simeq_{K \cdot h \cdot e}\left\{x_{0}\right\}$ for some point $x_{0} \in X$. Up to now, we have studied the notions of a $K$-homotopy and a $K$-homotopy equivalence and their properties. 
(a)

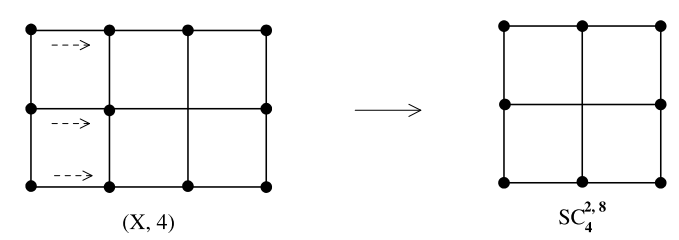

(b)

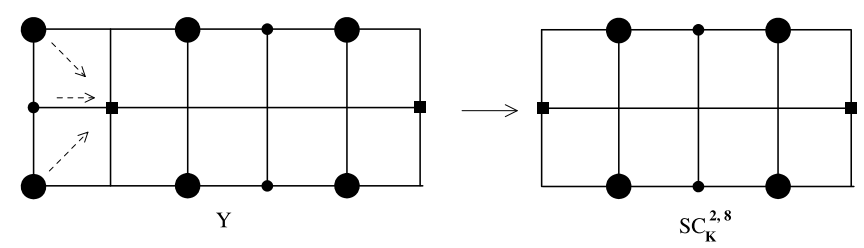

(c)

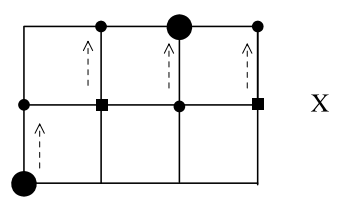

Figure 3 Explanation of both 4 - and $\boldsymbol{K}$-homotopies in digital topology. (a) A 4-homotopy in DTC (b) a K-homotopy in KTC; (c) comparison between an 8-homotopy in DTC and a K-homotopy in KTC.

Proposition 3.6 The k-homotopy equivalence in DTC and the K-homotopy equivalence in KTC have their own features, where the $k$-adjacency relation is taken from (2.2).

Proof Let us compare among two homotopies in terms of the pictures in Figure 3. We can see some intrinsic processes depending on the corresponding homotopies.

(1) In Figure 3(a), consider the digital image $(X, 4)$. By using the 4-homotopy, we see that $(X, 4)$ is 4-homotopy equivalent to $S C_{4}^{2,8}$.

(2) In Figure 3(b), consider the $K$-topological space $\left(Y, \kappa_{Y}^{2}\right)$. By using the $K$-homotopy we see that $\left(Y, \kappa_{Y}^{2}\right)$ is $K$-homotopy equivalent to $S C_{K}^{2,8}$.

\section{A relation between digital contractibilities and local contractibilities}

The notions of contractibility and locally contractibility play an important role in many areas of mathematics $[2,4,5,33]$. We say that a contractible space is precisely one with the same homotopy type of a singleton [33]. Furthermore, its digital versions have been developed in Definitions 4 and 7 in $D T C$ and $K T C$, respectively. In relation to the study of the conjecture (1.3), we need the following:

Definition 8 [7] A topological space $X$ is said to be locally contractible if it satisfies the following equivalent conditions:

(1) It has a basis of open subsets each of which is a contractible space under the subspace topology.

(2) For every $x \in X$ and every open subset $V$ ( $\ni x)$ of $X$, there exists an open subset $U$ ( $\ni x$ ) of $X$ such that $U \subset V$ and $U$ is a contractible space in the subspace topology derived from $V$.

In classical mathematics, it is well known that contractible spaces are not necessarily locally contractible nor vice versa [7]. For instance, whereas any $C W$-complex is locally 
contractible and any paracompact manifold is locally contractible [7], they need not be contractible,for example, the $n \mathrm{D}$ sphere $S^{n}, n \in \mathbf{N}$. Although the comb space [34] is contractible, it cannot be a locally contractible space. Besides, the cone on the Hawaiian earring space [34] is contractible, but it is not locally contractible.

To deal with the conjecture (1.3), we need to establish digital versions of local contractibilities in DTC and KTC. Motivated by the notion of local contractibility in Definition 8, let us establish their digital versions in $D T C$ and $K T C$.

\section{Definition 9}

(1) In DTC, a digital image $(X, k)$ is said to be locally $k$-contractible if every point $x \in X$ has an $N_{k}(x, 1)$ that is $k$-contractible.

(2) In $K T C$, a $K$-topological space $\left(X, \kappa_{X}^{n}\right)$ is said to be locally $K$-contractible if it has a basis of open subsets each of which is a $K$-contractible space under the subspace $K$-topology.

Let us recall the digital contractibility from the viewpoint of digital topology in a graphtheoretical approach. In $[9,11]$, the $k$-contractibility of some simple closed $k$-curves (see Figure 4) is proved. Namely, it turns out that $S C_{2 n}^{2 n, 4}$ is $2 n$-contractible [25] and, further, $S C_{3^{n}-1}^{n, 4}$ is $\left(3^{n}-1\right)$-contractible (in case $n=2$, see $[9,11]$, and in case $n \geq 3$, see [30]); see Figure 4 .

Proposition 4.1 Every digital space in DTC or KTC is locally contractible.

Proof (1) In DTC, since each point $x$ of a digital image $(X, k)$ has $N_{k}(x, 1)$ (see (2.4)) which is always $k$-contractible, the proof is completed.

(2) In $K T C$, each point $x$ of a $K$-topological space $\left(X, \kappa_{X}^{n}\right)$ has $\mathrm{SN}_{K}(x)$ (see (2.5)) which is $K$-contractible. To be specific, depending on the point $x \in \mathbf{Z}^{n}$, we have its smallest open neighborhood $\mathrm{SN}_{K}(x)$ (see (2.5) for the case of $\left(\mathbf{Z}^{2}, \kappa^{2}\right)$ ) that is $K$-contractible (see Figure 5). More precisely, based on Figure 5, consider the maps on $\operatorname{SN}_{K}(p)$ for the cases of

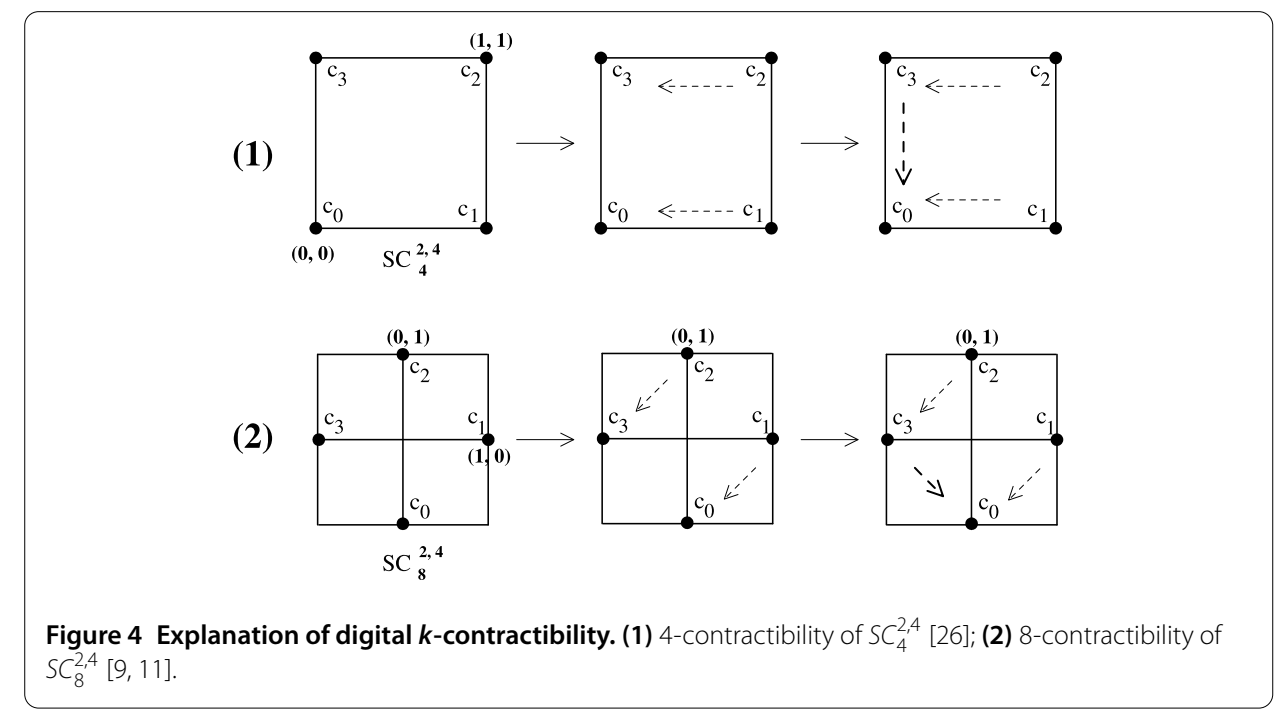


(1)

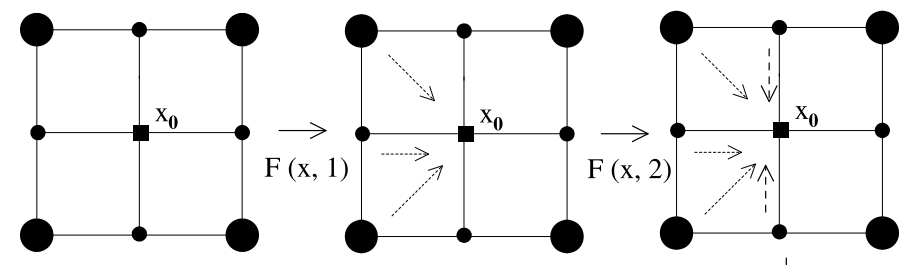

$\mathrm{F}(\mathrm{x}, 3) \downarrow$
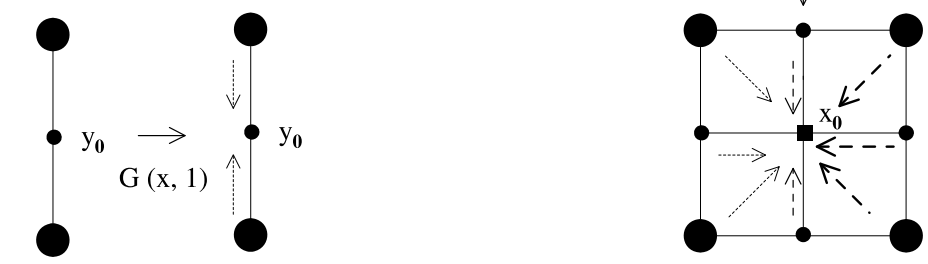

Figure 5 Examples for explaining local contractibility of the given two $K$-topological spaces (1) and (2).

$\left(\mathbf{Z}^{2}, \kappa^{2}\right):$

$$
\begin{aligned}
& F: \mathrm{SN}_{K}\left(x_{0}\right) \times[0,3]_{\mathrm{Z}} \rightarrow \mathrm{SN}_{K}\left(x_{0}\right) \text { shown in Figure 5(1) and } \\
& G: \mathrm{SN}_{K}\left(y_{0}\right) \times[0,1]_{\mathrm{Z}} \rightarrow \mathrm{SN}_{K}\left(y_{0}\right) \text { shown in Figure 5(2). }
\end{aligned}
$$

Then it is clear to see that the maps $F$ and $G$ are $K$-homotopies on $\operatorname{SN}_{K}\left(x_{0}\right)$ and $\operatorname{SN}_{K}\left(y_{0}\right)$, respectively. Furthermore, it is obvious that they make both $\operatorname{SN}_{K}\left(x_{0}\right)$ and $\operatorname{SN}_{K}\left(y_{0}\right) K$-contractible.

By using the method similar to the case of $\left(\mathbf{Z}^{2}, \kappa^{2}\right)$ we can prove the $K$-contractibility of $\mathrm{SN}_{K}(p)$ in $\left(\mathbf{Z}^{n}, \kappa^{n}\right)$.

Let us investigate some properties of $K$-contractibility in $K T C$.

Lemma 4.2 Any K-path in $\left(\mathbf{Z}^{n}, \kappa^{n}\right)$ is $K$-contractible.

Proof We will proceed in two steps.

Step 1. Let us consider a $K$-path in $\mathbf{Z}^{n}$, denoted by $X:=\left(x_{i}\right)_{i \in\left[0, l_{\mathbf{Z}}\right.}$, as a subspace induced by $\left(\mathbf{Z}^{n}, \kappa^{n}\right)$. Then it is obvious that $X$ contains a simple $K$-path $\left(x_{i}^{\prime}\right)_{i \in\left[0, l^{\prime}\right] \mathbf{Z}}:=X^{\prime} \subset X$ with $l^{\prime} \leq l$. If $X \backslash X^{\prime}$ is nonempty, then take $x_{j} \in X \backslash X^{\prime}$ such that $x_{j} \in \mathrm{SN}_{K}\left(x_{i}\right)$, where $x_{i} \in X^{\prime}$, that is, $x_{i}$ and $x_{j}$ are $K$-adjacent to each other. Then consider the map

$$
F:\left(X \times[a, a+1]_{\mathbf{Z}}, \kappa_{X \times[a, a+1]_{\mathbf{Z}}}^{n+1}\right) \rightarrow\left(X, \kappa_{X}^{n}\right)
$$

given by

$$
\left\{\begin{array}{l}
\text { (1) } F(x, a)=1_{X}, x \in X \\
\text { (2) } F\left(x^{\prime}, a+1\right)=1_{X^{\prime}}, x^{\prime} \in X^{\prime} \text {, and } \\
\quad \text { if } x_{j} \in X \backslash X^{\prime} \text { and } x_{j} \in \operatorname{SN}_{K}\left(x_{i}\right) \text {, then } x_{j} \rightarrow x_{i} .
\end{array}\right\}
$$

Then this map $F$ is a $K$-homotopy (see the process of $F(x, 1)$ in Figure 7 ). 
(a)

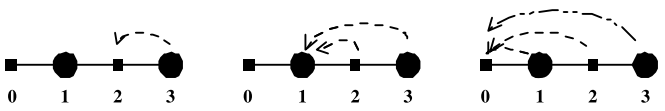

(1)

(2)

(3)

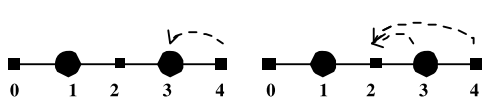

(1)

(2)

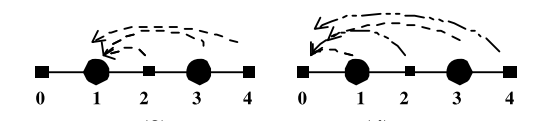

(3)

(4)

(b)
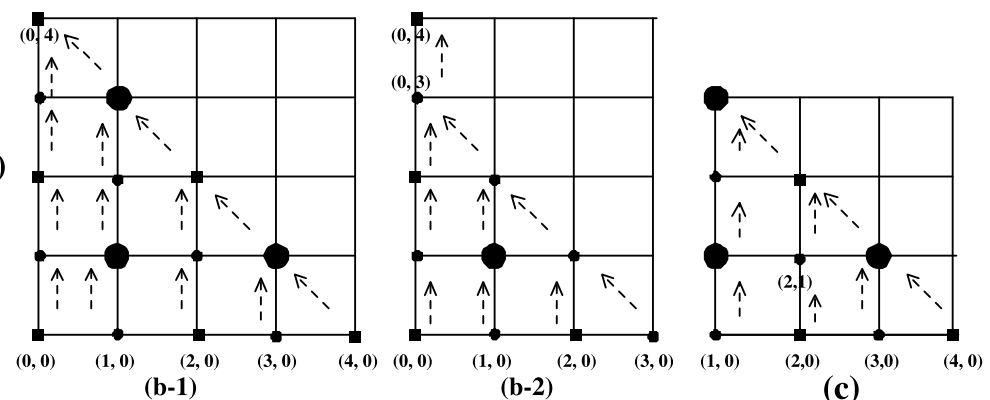

Figure 6 Explanation of $K$-contractibility of a $K$-path.

Step 2. Since $X^{\prime}$ is a simple $K$-path, by Proposition 3.4 we have a $K$-interval $\left([a, b]_{\mathrm{Z}}\right.$, $\left.\kappa_{[a, b]_{\mathbf{Z}}}\right)$ that is $K$-homeomorphic to $X^{\prime}:=\left(x_{i}^{\prime}\right)_{i \in[0, l]_{\mathbf{Z}}}$, where $\left([a, b]_{\mathbf{Z}}, \kappa_{[a, b]_{\mathbf{Z}}}\right)$ is $K$-homeomorphic to the subspace $\left([0, l]_{\mathrm{Z}}, \kappa_{[0, l]_{\mathrm{Z}}}\right)$ or $\left([1, l+1]_{\mathrm{Z}}, \kappa_{[1, l+1]_{\mathrm{Z}}}\right)$ where the cardinality of $[a, b]_{\mathrm{Z}}$ is equal to that of $[0, l]_{\mathbf{Z}}$ or $[1, l+1]_{\mathbf{Z}}$, that is, $b-a=l$. It is obvious that the $K$-contractibility of a simple $K$-path is equivalent to the $K$-contractibility of $\left([0, l]_{\mathrm{Z}}, \kappa_{\left[0, l l_{\mathrm{Z}}\right.}\right)$ or $\left([1, l+1]_{\mathbf{Z}}, \kappa_{[1, l+1]_{\mathbf{Z}}}\right)$. Hence, it suffices to prove that the identity map $1_{[0, l]_{\mathbf{Z}}}$ on $\left([0, l]_{\mathbf{Z}}, \kappa_{[0, l]_{\mathbf{Z}}}\right)$ is $K$-homotopic to the constant function $C_{\{0\}}$ given by $C_{\{0\}}(x)=0$ for all $x \in[0, l]_{Z}$ because the proof of the $K$-contractibility of $\left([1, l+1]_{\mathbf{Z}}, \kappa_{[1, l+1]_{\mathbf{Z}}}\right)$ is similar to that of $\left([0, l]_{\mathbf{Z}}, \kappa_{[0, l]_{\mathbf{Z}}}\right)$.

Since the number $l$ is finite, for some $m \in \mathbf{N}$ and any $s \in[0, l]_{\mathrm{Z}}$, define the map (see Figures 6(a) and 6(b))

$$
H:\left([0, l]_{\mathbf{Z}} \times[0, m]_{\mathbf{Z}}, \kappa_{[0, l]_{\mathbf{Z}} \times[0, m]_{\mathbf{Z}}}^{2}\right) \rightarrow\left([0, l]_{\mathbf{Z}}, \kappa_{[0, l]_{\mathbf{Z}}}\right)
$$

given by

$$
H(s, t)=\left\{\begin{array}{l}
1_{[0, l]_{\mathbf{Z}}}(s), t=0 ; \\
0, t \geqslant 0 \text { and } H(s, t-1)=0 ; \\
H(s, t-1)-1, t \geqslant 0 \text { and } H(s, t-1) \geqslant 0 .
\end{array}\right\}
$$

It is clear that $H$ is a $K$-homotopy between $1_{[0, l]_{Z}}$ and the constant map $C_{\{0\}}$, which is the trivial identity map on the singleton $\{0\}$.

For instance, let us consider the $K$-intervals $\left([0,3]_{\mathbf{Z}}, \kappa_{[0,3]_{\mathbf{Z}}}\right)$ and $\left([0,4]_{\mathbf{Z}}, \kappa_{[0,4]_{\mathbf{Z}}}\right)$ (see Figure 6(a)). Then, in terms of the process from (1) to (4) shown in Figures 6(a) and 6(b), the $K$-intervals $\left([0,3]_{\mathbf{Z}}, \kappa_{[0,3]_{\mathbf{Z}}}\right)$ and $\left([0,4]_{\mathbf{Z}}, \kappa_{[0,4]_{\mathbf{Z}}}\right)$ are proved to be $K$-contractible.

Concretely, combining Steps 1 and 2 , for some $m \in \mathbf{N}$, we obtain the map

$$
G:\left(X \times[0, m]_{\mathrm{Z}}, \kappa_{X \times[0,]_{\mathrm{Z}}}^{n+1}\right) \rightarrow\left(X, \kappa_{X}^{n}\right)
$$



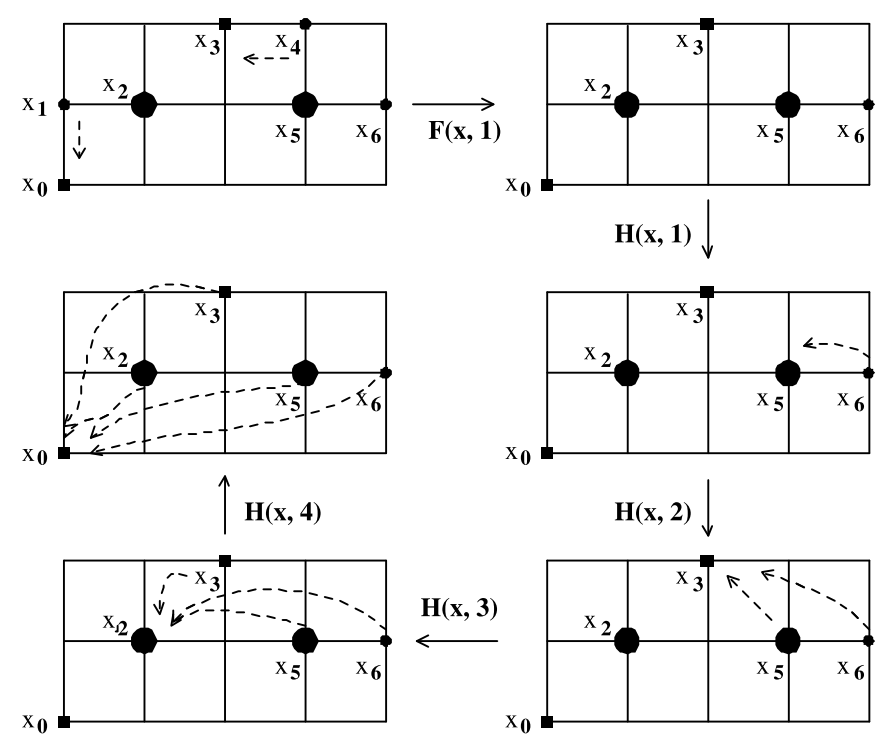

Figure 7 Explanation of $K$-contractibility of a $K$-path.

(a)

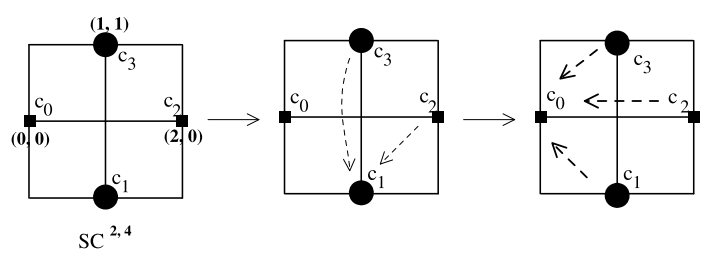

(b)

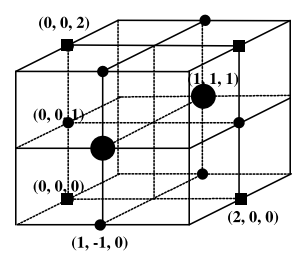

(1)

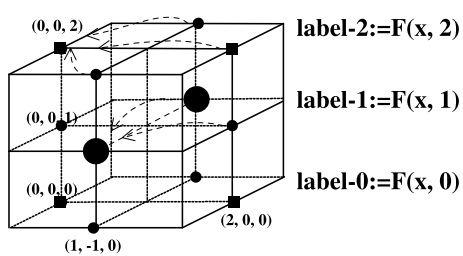

(2)

Figure 8 Explanation of $K$-contractibility of $S C_{K}^{2,4}$.

given by (see the process with combined $F(x, 1)$ and $H(x, i), i \in[1,4]_{\mathrm{Z}}$, in Figure 7 )

$$
\left\{\begin{array}{l}
G(x, t)=F(x, t), t \in\{0,1\} \text { and } \\
G(x, t) \simeq_{K} H(x, t), t \in[2, m]_{\mathbf{Z}} .
\end{array}\right\}
$$

Then we see that $G$ is a $K$-homotopy between $1_{\left(X, \kappa_{X}^{n}\right)}$ and $C_{\left\{x_{0}\right\}}$, which implies the $K$-contractibility of a $K$-path.

Lemma 4.3 $S C_{K}^{2,4}$ is $K$-contractible.

Proof The process presented in Figures 8(a) and 8(b) explains the following $K$-contractibility of $S C_{K}^{2,4}$. Motivated by Proposition 3.4(3), let us consider the map (see Figures 8(a) 
and $8(\mathrm{~b})(2))$

$$
F: S C_{K}^{2,4} \times[0,2]_{\mathbf{z}} \rightarrow S C_{K}^{2,4}
$$

such that

$$
\left\{\begin{array}{l}
\text { for all } x \in S C_{K}^{2,4}, F(x, 0)=1_{S C_{K}^{2,4}} ; \\
F(x, 1)=\left\{c_{1}\right\}, x \in\left\{c_{1}, c_{2}, c_{3}\right\}, F\left(c_{0}, 1\right)=\left\{c_{0}\right\} ; \text { and } \\
F(x, 2)=\left\{c_{0}\right\}, x \in S C_{K}^{2,4} .
\end{array}\right\}
$$

At this moment, in Figure 8(b)(1), we see that $S C_{K}^{2,4} \times\{0\} \simeq_{K} S C_{K}^{2,4} \times\{1\} \simeq_{K} S C_{K}^{2,4} \times$ $\{2\}$. Then it is obvious that the map $F$ (see (4.3)) is a $K$-homotopy supporting the $K$-homotopy equivalence between $S C_{K}^{2,4}$ and the singleton $\left\{c_{0}\right\}$, which implies that $S C_{K}^{2,4}$ is $K$-contractible.

By using the method given by (4.2) we obtain the following:

Corollary 4.4 A K-connected proper subset of $S C_{K}^{n, l}$ is K-contractible.

Proof By using the method similar to (4.2), we see that a $K$-connected proper subset of $S C_{K}^{n, l}$ is $K$-contractible.

Motivated by non- $k$-contractibility of $S C_{k}^{n, l}, l \geqslant 4$ [11], we obtain the following:

Lemma 4.5 $S C_{K}^{n, l}$ is not K-contractible if $l \geqslant 4$.

Proof Let us consider $S C_{K}^{2, l}, l \geqslant 4$ (see the spaces $W$ and $Z$ in Figure $9(\mathrm{~b})$ as $S C_{K}^{2,8}$ ). Then there is at least a part inside of $S C_{K}^{2, l}$ consisting of two points, a pure point and a mixed point, which are $K$-adjacent. Due to the part, there is no $K$-homotopy making $S C_{K}^{2, l}$ $K$-contractible.

By using the method similar to non- $K$-contractibility of $S C_{K}^{2, l}, l \geqslant 4$, we prove the non$K$-contractibility of $S C_{K}^{n, l}, l \geqslant 4$.

Theorem 4.6 The digital contractibility implies the local contractibility. The converse does not hold.

Proof Owing to Proposition 4.1, since every digital space is locally contractible, it suffices to prove that the local contractibility does not imply contractibility in DTC and KTC.

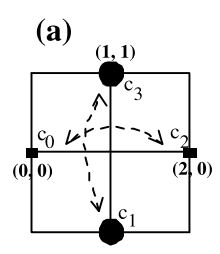

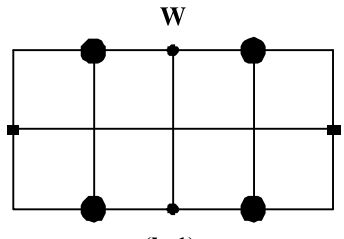

(b-1) (b)

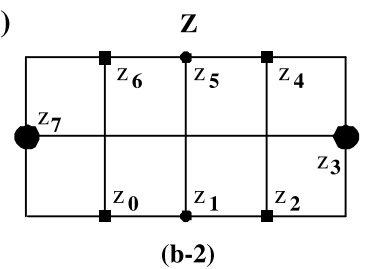

Figure 9 Explanation of the nonexistence of the FPP of a $K$-topological space. 
(1) In $D T C$, consider $S C_{k}^{n, l}$ such as $S C_{8}^{2,6}$ that is not $k$-contractible. By Proposition 4.1, whereas it is locally $k$-contractible, it is not $k$-contractible.

(2) In $K T C$, consider $S C_{K}^{n, l}$ such as $S C_{K}^{2,8}$ (see Figure 9(b)) that is not $K$-contractible. By Proposition 4.1, whereas it is locally $K$-contractible, it is not $K$-contractible.

\section{Contractibility and fixed point property: the case of Khalimsky topological spaces}

To study the FPP of digital spaces, we need to recall again that a digital space $X$ (resp. digital image $(X, k))$ is connected (resp. $k$-connected) and $|X| \geq 2$.

Rosenfeld [14] was the first to come up with a fixed point theorem of a digitally continuous self-map of a digital image $(X, k)$ in $\mathbf{Z}^{n}$ with the familiar Euclidean and city block distances. Besides, it was proved in [14] that any digital line segment $\left([a, b]_{\mathrm{Z}}, 2\right)$ does not have the $F P P$ from the viewpoint of digital topology in a graph-theoretical approach, where the cardinality of $[a, b]_{\mathbf{Z}}$ is greater than 1 , that is, $\left|[a, b]_{\mathbf{Z}}\right| \geq 2$. This property can be proved as follows. Take two distinct 2-adjacent points such as $x_{i}$ and $x_{j}$ in $\left([a, b]_{\mathrm{Z}}, 2\right)$. Then, for convenience, we may assume that $x_{i}$ is even and $x_{j}$ is odd. Consider the self-map $f$ of $\left([a, b]_{\mathrm{Z}}, 2\right)$, as follows: for any even numbers $x \in[a, b]_{\mathrm{Z}}, f(x)=x_{j}$, and the other odd numbers in $[a, b]_{\mathrm{Z}}$ are mapped by the map $f$ into the set $\left\{x_{i}\right\}$. Namely, the image $f\left([a, b]_{\mathrm{Z}}\right)$ has the cardinality 2 . Then it is clear that the given $\operatorname{map} f$ is a 2-continuous map that has no fixed points.

For the case of digital image $(X, 2 n)$ in $\mathbf{Z}^{n}$ with $|X| \geq 2$, using the method similar to the above approach, let us consider a $2 n$-continuous self-map $f$ of a digital image $(X, 2 n)$. Take two distinct points $x_{i}$ and $x_{j}$ that are $2 n$-adjacent in $X$. Let $f(x)=x_{i}, x \neq x_{i}$, and $f\left(x_{i}\right)=x_{j}$ [14]. Then we see that whereas the given map $f$ is a $2 n$-continuous map, it cannot have any fixed point. Similarly, Rosenfeld [14] proved that any digital image $(X, k)$ with $|X| \geq 2$ does not have the FPP either (see Proposition 5.1) as follows: take two $k$-adjacent points $x, y \in X$ in $\mathbf{Z}^{n}$ and consider a self-map $f$ of $(X, k)$ such that, for all $x_{1} \in X$ such that $x_{1} \neq x$,

$$
f\left(x_{1}\right)=x \text { and } f(x)=y
$$

Then, it is obvious that whereas the given map $f$ is a $k$-continuous map, it has no fixed points (for more details, see [24-26]).

Proposition 5.1 [14] (see Theorems 3.3 and 4.1 of [14]) A digital image $(X, k)$ in $\mathbf{Z}^{n}$ does not have the FPP if $X$ is $k$-connected and $|X| \geq 2$.

Motivated by the Lefschetz fixed point theorem in [3], Ege and Karaca [27] (Theorem 3.8 of [27]) studied a fixed point theorem of a $k$-continuous map on a $k$-contractible digital image in DTC as follows. Let $(X, k)$ be a digital image, and let $f:(X, k) \rightarrow(X, k)$ be any $k$-continuous map. If $(X, k)$ is $k$-contractible, then $f$ has a fixed point. However, by Proposition 5.1 it is clear that this assertion is incorrect [24-26]. Thus, by Proposition 5.1 we conclude the following:

Remark 5.2 [26] (see also [24-26]) The conjecture (1.3) is invalid in DTC.

To make the paper self-contained and to guarantee Remark 5.2, we have a very simple example: consider a bijective self-map of $\left([0,1]_{\mathbf{Z}}, 2\right)$ in $D T C$ such that $f(0)=1$ and $f(1)=0$ 
$[25,26]$; whereas $\left([0,1]_{\mathrm{Z}}, 2\right)$ is 2 -contractible in terms of the property $(4.2)$, from the viewpoint of DTC and further, the map $f$ is a 2-continuous map, which implies that $f$ cannot have any fixed point $[25,26]$.

Let us now move to the conjecture posed in (1.3).

Question In $K T C$, is the conjecture (1.3) valid?

We say that a $K$-topological space $\left(X, \kappa_{X}^{n}\right)$ has the $F P P$ if every $K$-continuous self-map $f$ of $X$ has a point $x \in X$ such that $f(x)=x$.

Let us now study some properties of $K$-topological spaces from the viewpoint of fixed point theory.

In $K T C$, we say that a $K$-topological invariant is a property of a $K$-topological space that is invariant under $K$-homeomorphisms.

Proposition 5.3 In KTC, the FPP is a K-topological invariant.

Proof Suppose that $\left(X, \kappa_{X}^{n_{0}}\right)$ has the FPP and there exists a $K$-homeomorphism $h$ : $\left(X, \kappa_{X}^{n_{0}}\right) \rightarrow\left(Y, \kappa_{Y}^{n_{1}}\right)$. Then we prove that $\left(Y, \kappa_{Y}^{n_{1}}\right)$ has the FPP. To this end, let $g$ be any $K$-continuous self-map of $\left(Y, \kappa_{Y}^{n_{1}}\right)$. Then consider the composition $h \circ f \circ h^{-1}:=g$ : $\left(Y, \kappa_{Y}^{n_{1}}\right) \rightarrow\left(Y, \kappa_{Y}^{n_{1}}\right)$, where $f$ is a $K$-continuous self-map of $\left(X, \kappa_{X}^{n_{0}}\right)$. Owing to the hypothesis, assume that $x \in X$ is a fixed point for a $K$-continuous self-map $f$ of $\left(X, \kappa_{X}^{n_{0}}\right)$. Since $h$ is a $K$-homeomorphism, there is a point $y \in Y$ such that $h(x)=y$. Let us consider the mapping

$$
f(x)=h^{-1} \circ g \circ h(x)=h^{-1}(g(h(x)))=h^{-1}(g(y)) .
$$

Then, from (5.2) we obtain $h(f(x))=g(y)$. Further, by the hypothesis of the $F P P$ of $\left(X, \kappa_{X}^{n_{0}}\right)$ and the $K$-homeomorphism between $\left(X, \kappa_{X}^{n_{0}}\right)$ and $\left(Y, \kappa_{Y}^{n_{1}}\right)$, we have

$$
h(f(x))=h(x)=y=g(y),
$$

which implies that the point $h(x)$ is a fixed point of the map $g$, which implies that $\left(Y, \kappa_{Y}^{n_{1}}\right)$ has the FPP.

Theorem 5.4 Let $X$ be a simple K-path in the $n D$ Khalimsky space. Then it has the FPP.

Proof In [35], it is proved that any bounded $K$-interval $\left([a, b]_{\mathrm{Z}}, \kappa_{[a, b] \mathrm{Z}}\right)$ has the FPP. Besides, by Proposition 3.4(1) it is obvious that any simple $K$-path in the $n \mathrm{D}$ Khalimsky space is $K$-homeomorphic to a certain $K$-interval $\left([a, b]_{\mathbf{Z}}, \kappa_{[a, b]_{\mathbf{Z}}}\right)$. By Proposition 5.3 we obtain the assertion.

Example 5.5 Consider the $K$-interval $\left([0,2]_{\mathrm{Z}}, \kappa_{[0,2] \mathrm{Z}}\right)$ and any $K$-continuous self-maps of $\left([0,2]_{\mathbf{Z}}, \kappa_{[0,2]_{\mathbf{Z}}}\right)$. Then there are only seven types of $K$-continuous self-maps of $\left([0,2]_{\mathrm{Z}}\right.$, $\left.\kappa_{[0,2] \mathbf{Z}}\right)$ among nine self-mappings. It is obvious that each of them has at least one fixed point.

Corollary 5.6 $S C_{K}^{n, l}$ does not have the FPP. 
Proof By the property of $S C_{K}^{n, l}:=\left(x_{i}\right)_{i \in[0, l-1]_{\mathbf{Z}}}$ we obtain that any two $K$-adjacent points such as $x_{i}, x_{i+1}(\bmod l), i \in[0, l-1]_{\mathrm{Z}}$, have the following property:

$$
\left\{\begin{array}{l}
x_{i} \in \mathrm{SN}_{K}\left(x_{i+1(\bmod l)) \text { or }}\right. \\
x_{i+1(\bmod l)} \in \mathrm{SN}_{K}\left(x_{i}\right) .
\end{array}\right\}
$$

In (5.3), in case $x_{i} \in \mathrm{SN}_{K}\left(x_{i+1}(\bmod l)\right.$ ), it is obvious that the cardinality of $\mathrm{SN}_{K}\left(x_{i+1}(\bmod l)\right)$ is three, and in case $x_{i+1}(\bmod l) \in \mathrm{SN}_{K}\left(x_{i}\right)$, we see that the cardinality of $\operatorname{SN}_{K}\left(x_{i}\right)$ is three. Thus, the number $l$ should be even and greater than or equal to 4 because these kinds of alternative arrangement of $x_{i}, x_{i+1}(\bmod l), i \in[0, l-1]_{\mathrm{Z}}$, are consecutive. Then consider the self-map $f$ of $S C_{K}^{n, l}$ given by $f\left(x_{i}\right)=x_{i+2}(\bmod l)$. Then it is clear that $f$ is a $K$-continuous map without any fixed point.

Example 5.7 Consider two types of $S C_{K}^{2,8}$ in Figures 9(b-1) and 9(b-2). Take the space $S C_{K}^{2,8}:=Z$ in Figure 9(b-2). Next, consider the self-map $f$ of $S C_{K}^{2,8}:=Z$ given by $f\left(z_{i}\right)=$ $z_{i+2}(\bmod 8)$. Whereas this map $f$ is obviously a $K$-continuous map, it has no fixed points (see $S C_{K}^{2,8}$ in Figures 9(b-1) and 9(b-2)).

Theorem 5.8 In KTC, the conjecture (1.3) is not valid.

Proof It suffices to propose a counterexample supporting this assertion. Let us consider $S C_{K}^{n, 4}, n \geq 2$, such as $S C_{K}^{2,4}$ (see Figure 9(a)), Then we see that $S C_{K}^{n, 4}, n \geq 2$, is $K$-homeomorphic to $S C_{K}^{2,4}$. Then, by Lemma 4.3 it is obvious that $S C_{K}^{n, 4}$ is $K$-contractible. Consider the self-map $f$ of $S C_{K}^{n, 4}$ given by

$$
f\left(c_{0}\right)=c_{2}, \quad f\left(c_{2}\right)=c_{0}, \quad f\left(c_{1}\right)=c_{3}, \quad f\left(c_{3}\right)=c_{1} .
$$

Whereas the map $f$ is obviously $K$-continuous map, it has no fixed points.

\section{Summary and further works}

Developing the notion of $K$-homotopy in the category of Khalimsky topological spaces, we have developed the notions of contractibility and local contractibility induced by the $K$-homotopy. Besides, proving that digital contractibilities imply local contractibilities for a $K$-contractible space $X$, we wondered if the space $X$ has the $F P P$. In this paper, we proved that not every $K$-topological space with $K$-contractibility has the FPP. More precisely, for $S C_{K}^{n, l}$, we proved that $S C_{K}^{n, l}$ does not have the $F P P$. For instance, we proved that whereas $S C_{K}^{n, 4}$ is $K$-contractible, it cannot have the $F P P$. However, we proved that a simple $K$-path has the $F P P$. In addition, we proved that in $K T C$ the $F P P$ is a $K$-topological invariant.

As a further work, we need to study the $F P P$ of the product of two simple $K$-paths. Besides, we need to study the FPP for other digital topological spaces. 


\section{References}

1. Schauder, J: Der Fixpunktsatz in Funktionalraumen. Stud. Math. 2, 171-180 (1930)

2. Borsuk, K: Theory of Retracts. Polish Sci., Warsaw (1967)

3. Lefschetz, S: Topology. Am. Math. Soc., New York (1930)

4. Cellina, A: A fixed point theorem for subsets of $L^{\prime}$. In: Multifunctions and Integrands. Lecture Notes in Mathematics, vol. 1091, pp. 129-137 (1984)

5. Fryszkowski, A: The generalization of Cellina's fixed point theorem. Stud. Math. 78, 213-215 (1984)

6. Ivashchenko, AV: Contractible transformations do not change the homology groups of graphs. Discrete Math. 126 , 159-170 (1994)

7. Mariconda, C: Contractibility and fixed point property: the case of decomposable sets. Nonlinear Anal., Theory Methods Appl. 18(7), 689-695 (1992)

8. Saha, PK, Chaudhuri, BB: A new approach to computing the Lefschetz number. Pattern Recognit. 28(12), 1955-1963 (1995)

9. Boxer, L: A classical construction for the digital fundamental group. J. Math. Imaging Vis. 10, 51-62 (1999)

10. Han, SE: On the classification of the digital images up to a digital homotopy equivalence. J. Comput. Commun. Res. 10, 194-207 (2000)

11. Han, SE: Non-product property of the digital fundamental group. Inf. Sci. 171(1-3), 73-91 (2005)

12. Kong, TY, Rosenfeld, A: Topological Algorithms for the Digital Image Processing. Elsevier, Amsterdam (1996)

13. Rosenfeld, A: Digital topology. Am. Math. Mon. 86, 76-87 (1979)

14. Rosenfeld, A: Continuous functions on digital pictures. Pattern Recognit. Lett. 4, 177-184 (1986)

15. Han, SE: Connected sum of digital closed surfaces. Inf. Sci. 176(3), 332-348 (2006)

16. Khalimsky, ED: Applications of connected ordered topological spaces in topology. In: Conference of Math. Department of Provoia (1970)

17. Han, SE: KD-(k, $\left.k_{1}\right)$-homotopy equivalence and its applications. J. Korean Math. Soc. 47(5), 1031-1054 (2010)

18. Smyth, MB, Tsaur, R: AFPP vs FPP: the link between almost fixed point properties of discrete structures and fixed point properties of spaces. Appl. Categ. Struct. 11, 95-116 (2003)

19. Khalimsky, E: Motion, deformation, and homotopy in finite spaces. In: Proceedings IEEE International Conferences on Systems, Man, and Cybernetics, pp. 227-234 (1987)

20. Han, SE: Continuities and homeomorphisms in computer topology and their applications. J. Korean Math. Soc. 45 , 923-952 (2008)

21. Han, SE, Yao, W: Homotopy based on Marcus Wyse topology and their applications. Topol. Appl. 201, 358-371 (2016)

22. Wyse, F, Marcus, D, et al.: Solution to problem 5712. Am. Math. Mon. 77, 1119 (1970)

23. Han, SE: Strong k-deformation retract and its applications. J. Korean Math. Soc. 44(6), 1479-1503 (2007)

24. Boxer, L, Ege, O, Karaca, I, Lopez, J: Digital fixed points, approximate fixed points, and universal functions. arXiv:1507.02349

25. Han, SE: Digital version of the fixed point theory. In: Proceedings of 11th ICFPTA (Abstracts), p. 60 (2015)

26. Han, SE: Fixed point theorems for digital images. Honam Math. J. 37(4), 595-608 (2015)

27. Ege, O, Karaca, I: Lefschetz fixed point theorem for digital images. Fixed Point Theory Appl. 2013, 253 (2013). doi:10.1186/1687-1812-2013-253

28. Han, S-E: Banach fixed point theorem from the viewpoint of digital topology. J. Nonlinear Sci. Appl. 9(3), 895-905 (2016)

29. Herman, GT: Oriented surfaces in digital spaces. CVGIP, Graph. Models Image Process. 55, 381-396 (1993)

30. Han, SE: The $k$-homotopic thinning and a torus-like digital image in $\mathbf{Z}^{n}$. J. Math. Imaging Vis. 31(1), 1-16 (2008)

31. Alexandorff, P: Diskrete Räume. Mat. Sb. 2, 501-518 (1937)

32. Han, SE, Park, BG: Digital graph $\left(k_{0}, k_{1}\right)$-homotopy equivalence and its applications. http://atlas-conferences.com/c/a/k/b/35.htm (2003)

33. Spanier, EH: Algebraic Topology. McGraw-Hill, New York (1966)

34. Munkres, JR: Topology. Prentice Hall, New York (1975)

35. Samieinia, S: The number of Khalimsky-continuous functions between two points. In: Combinatorial Image Analysis. Lecture Notes in Computer Science, vol. 6636, pp. 96-106 (2011)

\section{Submit your manuscript to a SpringerOpen ${ }^{\circ}$ journal and benefit from:}

- Convenient online submission

Rigorous peer review

- Immediate publication on acceptance

- Open access: articles freely available online

- High visibility within the field

- Retaining the copyright to your article 